\title{
Síndrome de hiperestimulación ovárica
}

\section{Ovarian hyperstimulation syndrome}

\author{
B. Azcona, G. Campo, J. Zabaleta
}

\section{RESUMEN}

La experiencia con el tratamiento inductor de la ovulación y el conocimiento de la fisiopatología del síndrome de hiperestimulación ovárica (SHO), los factores de riesgo y las características clínicas son claves para la prevención y el manejo del SHO. Las manifestaciones leves del SHO son bastante comunes, ocurriendo en hasta un tercio de los ciclos con superovulación inducidos por gonadotropinas exógenas.

El empeoramiento de los síntomas del SHO normalmente todavía se puede manejar de forma ambulatoria, pero es esencial la monitorización y evaluación frecuente. La enfermedad grave resultante del SHO es mucho menos común, pero puede conllevar riesgo vital. La hospitalización puede ser necesaria, en algunos casos.

Palabras clave. Síndrome de hiperestimulación ovárica. Inducción de la ovulación. Técnicas de reproducción asistida. Síndrome de ovario poliquístico. Fertilización in vitro. Gonadotropinas.

\begin{abstract}
Experience with ovulation induction treatment and understanding of the physiopathology of ovarian hyperstimulation syndrome (OHS), the risk factors and the clinical characteristics are of key importance for preventing and managing OHS. Light manifestations of OHS are fairly common, occurring in up to a third of the cycles with superovulation induced by exogenous gonadotropins.
\end{abstract}

A worsening of the symptoms of OHS can still normally be managed in out-patient form, but frequent monitoring and evaluation are essential. Serious disease resulting from OHS is much less common, but can involve risk to life. Hospitalisation might be necessary in some cases.

Key words. Ovarian hyperstimulation syndrome. Controlled ovarian hyperstimulation. Ovulation induction. Assisted reproductive technologies. Polycystic ovarian syndrome. In vitro fertilization. Gonadotropins.
Servicio de Obstetricia y Ginecología. Hospital Virgen del Camino. Pamplona.

\section{Correspondencia:}

Beatriz Azcona Ruiz

Servicio de Obstetricia y Ginecología

Hospital Virgen del Camino

Irunlarrea, 4

31008 Pamplona

Tfno. 625230168

E-mail: beatrizazconar@hotmail.com 


\section{INTRODUCCIÓN}

El síndrome de hiperestimulación ovárica $(\mathrm{SHO})$ es una respuesta exagerada del ovario a los tratamientos de estimulación ovárica. Se asocia de forma característica con la administración exógena de gonadotropinas y, más raramente, con citrato de clomifeno. Su incidencia en los ciclos de fecundación in vitro (FIV) varía entre el $0,6 \%$ y el $10 \%^{1}$.

El SHO se caracteriza por un amplio espectro clínico que varía desde formas leves asintomáticas en las que sólo existe un aumento del tamaño ovárico a formas graves con compromiso hemodinámico severo por extravasación de líquido al tercer espacio. La incidencia de las formas graves oscila entre $0,25-1,8 \%^{2}$.

La Sociedad Española de Ginecología y Obstetricia (SEGO) ha propuesto como índice de calidad la incidencia del síndrome de hiperestimulación grave en menos del $1 \%$ de los ciclos estimulados ${ }^{1}$.
El hecho de que pacientes jóvenes y sanas puedan desarrollar complicaciones potencialmente letales (fallo renal, fallo hepático, shock hipovolémico, fenómenos tromboembólicos, síndrome de distréss respiratorio del adulto) supone un problema médico de gran trascendencia.

También se han descrito algunos casos en ciclos naturales no estimulados: en casos de embarazos molares, adenomas productores de gonadotropinas, mutaciones en el receptor de FSH.

\section{FISIOPATOLOGÍA}

El fenómeno cardinal del cuadro es el aumento de la permeabilidad capilar que induce el desplazamiento de líquido y proteínas del espacio intravascular al tercer espacio (Fig. 1). Esto provoca una depleción del volumen intravascular responsable de los síntomas del síndrome: hipotensión, oliguria, ascitis, aumento de la viscosidad sanguínea, hiponatremia e hiperkaliemia.

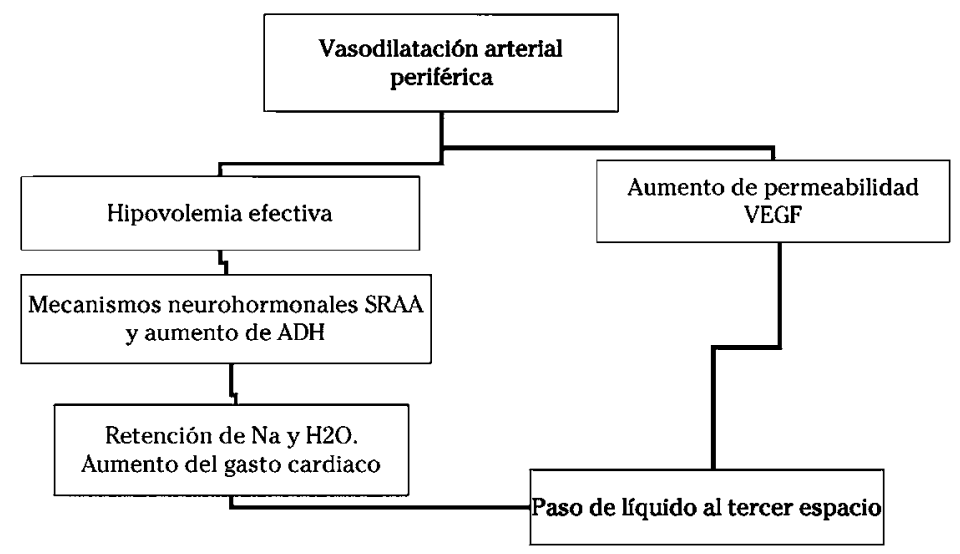

Figura 1. Fisiopatología de la hiperestimulación ovárica.

El aumento de la permeabilidad capilar se debe a la liberación de varias sustancias vasoactivas a la circulación sistémica. El principal agente es el factor de crecimiento endotelial vascular (VEGF) de origen folicular, aunque también se han implicado otros factores: IL-6, cascada de renina-angiotensina, prostaglandinas, sistema de quininacalicreína, estradiol... ${ }^{3}$
Estos mediadores se liberan tras la luteinización masiva de los folículos provocada por la hormona coriónica gonadotrofica (hCG)

El VEGF folicular tiene tres acciones principales que favorecen el aumento de la permeabilidad capilar ${ }^{2}$ :

1. Es un potente promotor de neoangiogénesis. 
2. Altera la funcionalidad e integridad de la pared vascular.

3. Altera las uniones intercelulares induciendo cambios en las fibras de actina.

\section{FACTORES DE RIESGO}

Todas las mujeres sometidas a un tratamiento de fertilidad tienen riesgo de sufrir una hiperestimulación ovárica ${ }^{4,5}$. Sin embargo hay unas más susceptibles que otras, por lo que el conocimiento de los factores de riesgo es útil para su prevención. Los factores de riesgo más comunes son:

- Edad joven (menos de 30 años): responden más a las gonadotropinas ya que tienen mayor densidad de receptores y más número de folículos susceptibles de responder a éstas.

- Bajo peso corporal: algunos autores encontraron una incidencia mayor de SHO en pacientes con bajo índice de masa corporal, aunque posteriormente otros no han hallado los mismos resultados.

- Síndrome de ovario poliquístico (SOP): el hiperandrogenismo, el hiperinsulinismo y el cociente $\mathrm{LH} / \mathrm{FSH}>2$ son condiciones que predisponen a la respuesta exagerada y a la aparición del cuadro.

- Imagen ovárica en la ecografía: “ovarios en collar".

- Niveles elevados de concentración de estradiol inmediatamente antes de la administración de la hCG o incrementos bruscos de los niveles previos a administrar la misma. Los valores de riesgo no están claramente establecidos, pero se puede considerar que el riesgo está muy aumentado con niveles de estradiol $>2.500 \mathrm{pg} / \mathrm{ml}$.

- Formación de un número elevado de folículos de tamaño intermedio (10-14 $\mathrm{mm})$.

- Instauración del embarazo y mayor riesgo si éste es múltiple.

- Mujeres que han desarrollado otras hiperestimulaciones en ciclos previos.

- Uso de hCG como soporte de fase lútea en lugar de progesterona.
- Alérgicas: la fisiopatología es similar por la respuesta inflamatoria que se produce en ambos cuadros (liberación de citoquinas e inmunomoduladores). En un estudio se observó un aumento significativo de la incidencia en las alérgicas respecto al grupo control ( 50 vs $21 \%$ ), sin embargo son precisos más estudios.

- Pico endógeno de LH: la hiperestimulación grave muy raramente se puede producir tras el pico endógeno de LH por un desarrollo espontáneo o farmacológico de múltiples folículos, aún sin administrar la hCG. Se han descrito casos en mujeres con ovario poliquístico o hipotiroidismo.

\section{CARACTERÍSTICAS CLÍNICAS ${ }^{6,7}$}

La sintomatología inicial se manifiesta entre los 3 y 10 días siguientes a la administración de la hCG pudiendo empeorar de forma rápida en caso de embarazo, debida a la producción endógena de $ß$-hCG por el trofoblasto. En caso de embarazo el cuadro persiste hasta que descienden los títulos de $ß-h C G$ a los 60-70 días de gestación. Por lo tanto puede ser útil la división del cuadro según el momento de aparición en:

- SHO temprano: Ocurre en los nueve días posteriores a la administración de la hCG. Es autolimitado (si no hay gestación) y de menor severidad.

- SHO tardío: Inicio a partir del $10^{\circ}$ día de administración de la hCG. Su causa es la secreción endógena de beta hCG producida por una gestación incipiente. Es un cuadro, en general, más severo y prolongado en el tiempo.

Las manifestaciones leves del SHO son relativamente comunes e incluyen: disconfort abdominal bajo transitorio, náuseas, vómitos y/o diarrea, distensión abdominal.

Existe enfermedad grave cuando el dolor se acompaña de uno o más de los siguientes signos:

- Ganancia rápida de peso.

- Ascitis a tensión.

- Inestabilidad hemodinámica (hipotensión ortostática, taquicardia...).

- Dificultad respiratoria (taquipnea).

- Oliguria progresiva. 
- Alteraciones de las pruebas de laboratorio.

Las complicaciones vitales que pueden darse son:

- Fracaso renal agudo.

- Hemorragia por rotura ovárica.

- Síndrome de distress respiratorio del adulto (SDRA).

- Tromboembolismo.

\section{MANEJO DIAGNÓSTICO- TERAPÉUTICO $^{6,7}$}

Junto con la clínica, que es la base del diagnóstico, la confirmación exige la realización de una ecografía pélvica (Fig. 2) para valorar el tamaño de los ovarios y la ascitis, además en algunos casos se pueden necesitar otras pruebas complementarias que ayuden a estadiar la gravedad del proceso y a controlar su evolución.

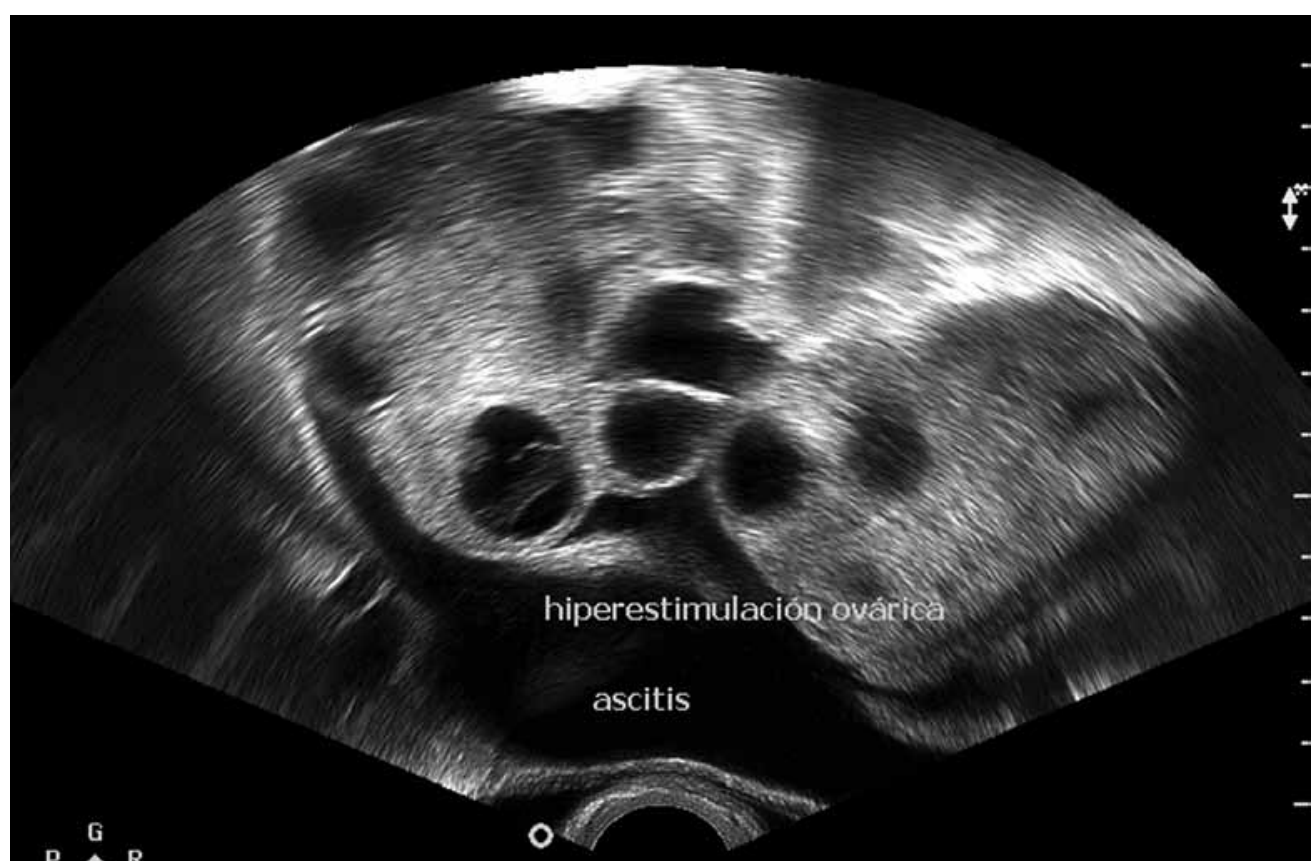

Figura 2. Ecografía de la hiperestimulación ovárica.

\section{Manejo ambulatorio}

Las pacientes con manifestaciones leves del SHO pueden ser manejadas de forma ambulatoria. El tratamiento normalmente requiere solo analgésicos orales y asesoramiento sobre los signos y síntomas de progresión de la enfermedad (Tabla 1). Es mejor evitar las relaciones sexuales ya que pueden ser dolorosas y pueden incrementar el riesgo de rotura ovárica.

El tratamiento del empeoramiento del SHO típicamente requiere antieméticos y analgésicos más potentes. La mayoría de las pacientes todavía se pueden manejar y monitorizar con efectividad de forma am- bulatoria pero requieren una evaluación más cuidadosa que incluya:

- Frecuentes exámenes físicos y ecográficos (para detectar ascitis progresiva).

- Medición diaria del peso.

- Determinaciones de laboratorio seriadas del hematocrito, electrolitos y creatinina sérica.

Las recomendaciones para el manejo ambulatorio del SHO persistente o que empeora incluyen:

- El aporte de líquido oral se debería mantener a no menos de 1 litro al día; cualquiera de las bebidas suplemen- 
tadas con electrolitos disponibles comercialmente es preferible a las otras bebidas.

- Se debe evitar la actividad física intensa ya que el riesgo de torsión ovárica aumente cuando los ovarios están significativamente agrandados. Se debe mantener una leve actividad física en la medida de lo posible. No es recomendable el reposo estricto en cama y puede incrementar el riesgo de tromboembolismo.
- El peso se debe registrar de forma diaria, así como la frecuencia y/o volumen de la orina. La ganancia de peso de $\geq 1 \mathrm{~kg}$ al día o la disminución de la frecuencia urinaria deberían promover la repetición del examen físico, ecografía y la evaluación de pruebas de laboratorio que incluyan hematocrito, electrolitos y creatinina sérica.

- Las pacientes embarazadas con SHO deberían se monitorizadas muy de cerca debido al riesgo de progresión de la enfermedad

Tabla 1. Estadiaje $\mathrm{e}^{7}$

\begin{tabular}{|c|c|}
\hline Grado & Síntomas \\
\hline $\begin{array}{l}\text { SHO leve } \\
\text { Grado I }\end{array}$ & $\begin{array}{l}\text { Distensión abdominal } \\
\text { Dolor abdominal leve } \\
\text { Tamaño ovárico habitualmente menor de } 8 \mathrm{~cm} .{ }^{*}\end{array}$ \\
\hline $\begin{array}{l}\text { SHO moderado } \\
\text { Grado II }\end{array}$ & $\begin{array}{l}\text { Dolor abdominal moderado } \\
\text { Náuseas, vómitos y/o diarrea } \\
\text { Evidencia ecográfica de ascitis } \\
\text { Tamaño ovárico habitualmente de } 8-12 \mathrm{~cm} .{ }^{*}\end{array}$ \\
\hline $\begin{array}{l}\text { SHO grave } \\
\text { Grado III }\end{array}$ & $\begin{array}{l}\text { Ascitis clínica, frecuentemente con hidrotórax } \\
\text { Oliguria con aumento de la creatinina } \\
\text { Hemoconcentración (Hto. > } 45 \% \text { o incremento de }>30 \% \text {, respecto al valor previo) } \\
\text { Hiponatremia } \\
\text { Trastornos de la coagulación } \\
\text { Trastornos de la función hepática (apreciables en el } 25-40 \% \text { de los casos) } \\
\text { Tamaño ovárico habitualmente mayor de } 12 \mathrm{~cm} .^{*}\end{array}$ \\
\hline SHO crítico & $\begin{array}{l}\text { Ascitis a tensión o hidrotórax severo } \\
\text { Hematocrito }>55 \% \\
\text { Leucocitosis }>25.000 / \mathrm{ml} \\
\text { Fallo renal. Creatinina }>1,6 \mathrm{mg} / \mathrm{dl} \text {. } \\
\text { Accidente tromboembólico } \\
\text { Síndrome de distress respiratorio del adulto }\end{array}$ \\
\hline
\end{tabular}

* El tamaño ovárico puede no estar relacionado con la severidad del SHO en los casos de reproducción asistida debido al efecto de la aspiración folicular.

\section{Hospitalización}

Dado el espectro y severidad de síntomas, así como el potencial de complicaciones, las mujeres con SHO grave deben ser hospitalizadas para una monitorización más cuidadosa y un tratamiento más agresivo.

Ningún síntoma o signo es una indicación absoluta, pero se debería considerar la hospitalización cuando está presente uno o más de los siguientes hallazgos ${ }^{7}$.
- Dolor abdominal severo o signos de irritación peritoneal.

- Náuseas intratables y vómitos que impiden la ingesta de comidas y líquidos adecuados.

- Oliguria severa o anuria.

- Ascitis a tensión.

- Disnea o taquipnea.

- Hipotensión (en relación a la basal), mareo o síncope. 
- Desequilibrio electrolítico severo (hiponatremia, hiperkaliemia).

- Hemoconcentración.

- Pruebas de función hepáticas anormales.

Los hallazgos de laboratorio en las mujeres con SHO grave incluyen:

- Hemoconcentración (hematocrito $>45 \%$ ).

- Leucocitosis (recuento > 15.000).

- Desequilibrio electrolítico (hiponatremia: sodio < $135 \mathrm{mEq} / \mathrm{L}$; hiperkaliemia: potasio $>5.0 \mathrm{mEq} / \mathrm{L}$ ).

- Enzimas hepáticos elevados.

- Disminución del aclaramiento de creatinina (creatinina sérica $>1,2$; aclaramiento de creatinina $<50 \mathrm{ml} / \mathrm{min}$ ).

Las recomendaciones para la evaluación y monitorización de las pacientes hospitalizadas con SHO incluyen examen de:

- Signos vitales (cada 2-8 horas).

- Peso (registro diario).

- Examen físico completo (diario, evitando el examen bimanual del los ovarios debido al riesgo de rotura ovárica).

- Circunferencia abdominal (a la altura del ombligo, registrada a diario).

- Monitorización de la entrada y salida de líquidos (diario, o más a menudo si es necesario).

- Examen ecográfico (ascitis, tamaño ovárico), repetido según sea necesario para guiar el manejo o la paracentesis.

- Radiografía de tórax y ecocardiografía (cuando se sospecha derrame pleural o pericárdico), repetido según sea necesario.

- Pulsioximetría (para las pacientes con síntomas de compromiso pulmonar)

- Recuento hemático completo (diario, o más a menudo según sea necesario para guiar el manejo de fluidos).

- Electrolitos (diario).

- Creatinina sérica o aclaramiento de creatinina, peso específico de la orina, repetido según sea necesario.

- Enzimas hepáticas, repetidas según sea necesario.
Es esencial la cuidadosa y frecuente reevaluación de la paciente hospitalizada con SHO severo. La queja de aumento del dolor y de la distensión abdominal obliga a una atención inmediata, teniendo en mente que el dolor y la ascitis fácilmente pueden enmascarar la rotura ovárica y la hemorragia intra-abdominal aguda. Las evaluaciones clínicas y de laboratorio seriadas aportan los medios para monitorizar la progresión de la enfermedad, para juzgar la respuesta al tratamiento y para reconocer la evidencia de la resolución.

\section{Manejo de los fluidos}

Las pacientes hospitalizadas requieren manejo con fluidos intravenosos (Fig. 3) para contrarrestar el incremento de la permeabilidad vascular que acompaña al SHO severo. Las guías para el manejo de fluidos para las pacientes hospitalizadas con enfermedad severa incluyen las siguientes:

- La monitorización estricta del aporte de líquidos y emisión de orina es esencial hasta que mejoran los síntomas o comienza la diuresis.

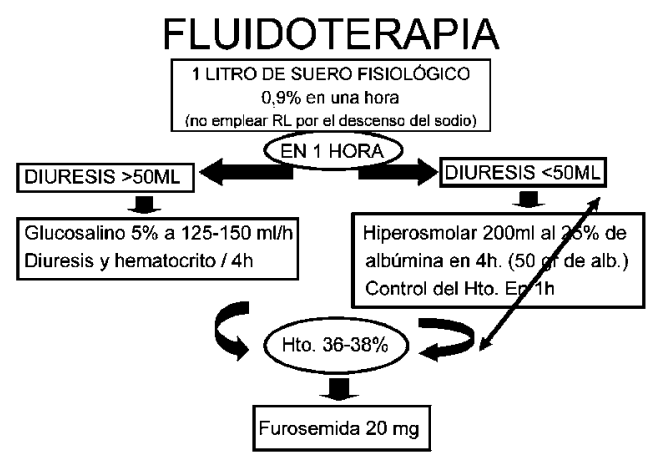

Figura 3. Tratamiento: fluidoterapia.

- La ingesta oral de líquidos debería estar registrada cuidadosamente y limitada a las cantidades necesarias para el confort de la paciente.

- Es conveniente hidratar adecuadamente a la paciente mediante un tratamiento inicial con 1 litro de suero salino intravenoso administrado en una hora. Se evaluará la diuresis en la hora siguiente y se definirá la pauta a seguir en función de ella (normal $>50$ $\mathrm{ml} / \mathrm{h}$ ), con determinación de la excre- 
ción urinaria y del hematocrito cada 4 horas.

- Si se restablece la diuresis se continuará con sueroterapia, en los volúmenes necesarios para mantener una producción adecuada de orina $(>20-30$ $\mathrm{ml} / \mathrm{h}$ ) y para revertir la hemoconcentración. Es preferible la dextrosa al 5\% en suero salino normal a la solución de Ringer lactato, dada la tendencia a al hiponatremia. La corrección de la hipovolemia, hipotensión y oliguria tiene la máxima prioridad, aún aceptando que la administración de fluidos puede contribuir al acúmulo de la ascitis.

- Si no existe respuesta al bolo de suero salino inicial se debe pautar un régimen hiperosmolar: el expansor del plasma más usado es la albúmina, aunque pueden ser usados otros (p.ej. hidroxietilalmidón 6\%, manitol, plasma fresco congelado). El dextrano se ha asociado con el desarrollo de SDRA por lo que es mejor que sea evitado. La pauta más habitual es albúmina IV (al 25\%) en dosis de 50-100 $\mathrm{g}$, con perfusión lenta en unas 4 horas y repetida a intervalos de 4 a 12 horas según sea necesario ${ }^{8}$.

- Se pueden considerar el tratamiento con diuréticos (p.ej. furosemida, 20 mg IV) pero no antes de que se haya restaurado el volumen vascular de forma adecuada (hematocrito $<38 \%$ ), ya que el uso prematuro o exagerado de diuréticos puede agravar la hipovolemia y la hemoconcentración, incrementando por lo tanto el riesgo de tromboembolia.

- Cuando el síndrome se está resolviendo, lo que se anuncia por la mejoría de los síntomas y la aparición de una diuresis eficiente, la administración de líquidos intravenosos debería restringirse rápidamente e incrementarse el aporte oral de fluidos.

- La hiperkaliemia está asociada con riesgo de arritmias cardiacas. Hay que tener precaución con la administración de sustancias que movilizan el potasio intracelular (insulina y glucosa, bicarbonato). Las manifestaciones electrocardiográficas de la hiperkaliemia (intervalos PR y QRS prolongados, depresión del segmento $\mathrm{ST}$, ondas $\mathrm{T}$ altas y picudas) indican la necesidad de tratamiento inmediato con gluconato cálcico.

\section{Paracentesis}

La paracentesis guiada por ecografía puede estar indicada para las pacientes con:

- Ascitis que causa dolor.

- Función pulmonar comprometida (p. ej. taquipnea, hipoxia, hidrotórax).

- Oliguria/anuria que no mejora con el manejo de fluidos apropiado.

Se puede usar la vía transvaginal o abdominal, bajo control ecográfico cuidadoso. El volumen óptimo de líquido que debe ser extraído en una sola sesión y el intervalo de tiempo en que se realiza, no está bien establecido. No obstante, es prudente extraer líquido a un ritmo deliberado hasta que se consigue el efecto deseado, mientras que se monitoriza cuidadosamente la respuesta de la paciente. Pueden ser requeridas paracentesis repetidas para mantener la función renal y pulmonar adecuadas.

La ascitis severa puede estar asociada con hidrotórax, más frecuentemente en el lado derecho, producto de la transferencia del líquido abdominal al tórax a través del conducto torácico. La paracentesis normalmente será efectiva en la resolución del hidrotórax y la toracocentesis debe estar reservada para los casos con derrames bilaterales o severos que persisten.

\section{Manejo de las complicaciones}

- Fallo renal: cuando todas las medidas usadas hayan fracasado, la perfusión de dopamina mejora significativamente la función renal $(0,18 \mathrm{mg} / \mathrm{kg} / \mathrm{h})$. Puede ser necesario la monitorización de la presión venosa central o la presión de enclavamiento en capilares pulmonares e incluso la diálisis a corto plazo.

- Complicaciones tromboembólicas: la incidencia de trombosis se sitúa entre 0,7 y $10 \%$ en las formas severas, por lo que están justificadas las medidas profilácticas. Se recomienda el uso de 
medias largas de compresión venosa y se debe considerar seriamente la profilaxis con heparina (5.000 UI SC cada 12 horas). El uso de un dispositivo de compresión neumática intermitente es prudente cuando los síntomas impiden la deambulación y confinan a la paciente en la cama. Los signos y síntomas sugestivos de tromboembolismo exigen la realización precoz de medidas diagnósticas adicionales (valoración de gases en sangre arterial, scanner de ventilación perfusión) y anticoagulación terapéutica cuando el diagnóstico se confirma o hay una alta sospecha.

- SDRA: el cuidado pulmonar intensivo puede incluir la suplementación con oxígeno, la toracocentesis y, cuando las medidas más conservadoras fallan, la ventilación asistida.

- Ruptura de quistes ováricos: el descenso del hematocrito, sin regresión de otros signos de $\mathrm{SHO}$, es indicador importante de hemorragia intraperitoneal. La cirugía debe ser conservadora al máximo.

- Torsión anexial: se produce en casos de SHO leve cuando los quistes son pequeños. Es difícil distinguir entre una torsión y la sensibilidad abdominal asociada a los ovarios estimulados. La aparición repentina de dolor junto a la existencia de un diámetro de ovario relativamente pequeño ayudan al diagnóstico. El Doppler para valorar la interrupción del flujo ovárico es de gran ayuda. La torsión ovárica se produce en el $26 \%$ de las mujeres que quedan gestantes y en el $2,3 \%$ de las no gestantes.

- Aparición de infecciones: (mayoritariamente urinarias o respiratorias) ya que en estas pacientes encontramos cierto nivel de inmunodepresión por descenso de inmunoglobulinas (incluyendo Ig G e Ig A), aumentando así la probabilidad de sufrir infecciones nosocomiales. Precisarán tratamiento antibiótico específico.

- La disfunción hepática también es frecuente en estas pacientes, su etiología es desconocida y no hay un tratamiento específico, sino que cura con la mejoría general del proceso.

\section{PREVENCIÓN}

Las claves para la prevención del SHO son la experiencia con la terapia de inducción de la ovulación y el reconocimiento de los factores de riesgo para el SHO. Los regímenes de inducción de la ovulación deberían ser altamente individualizados, monitorizados cuidadosamente y usando dosis y duración mínimas del tratamiento con gonadotropinas para conseguir la meta terapéutica ${ }^{9,10}$.

Hay que tener precaución cuando está presente cualquier de los siguientes indicadores de riesgo incrementado de SHO:

- Elevación rápida de los niveles de E2.

- Concentración de E2 mayor de 2.500 $\mathrm{pg} / \mathrm{ml}$.

- Aparición de un gran número de folículos de tamaño intermedio (10-14 $\mathrm{mm})$.

Existen distintas medidas para tratar de disminuir la incidencia de $\mathrm{SHO}^{10,11}$ :

1. Cancelación del ciclo y supresión de la administración de HCG: es la forma más eficaz de evitar el síndrome de hiperestimulación ovárica (los límites para administrar la hCG no están claramente establecidos pero se pueden situar en niveles de estradiol $>4.000 \mathrm{pg} / \mathrm{ml} \mathrm{o}>2.000$ $\mathrm{pg} / \mathrm{ml}$, con más de 15 folículos cada uno de ellos mayor de $12 \mathrm{~mm}$ ).

2. Disminuir la dosis de hCG a 5.000, en vez de las 10.000 UI que se emplean de forma rutinaria.

3. Criopreservación de embriones y su uso en otro ciclo: no se evita la hiperestimulación precoz, pero sí la tardía.

4. Apoyo de fase lútea con progesterona, no hCG.

5. Maduración final con un bolo de agonistas: alternativa útil para las pacientes tratadas previamente con antagonistas.

6. Administración profiláctica de albúmina al $20 \%$ o hidroxietilalmindón 6\%: la albúmina no ha demostrado su utilidad, además no está exenta de riesgos ya que puede producir reacciones febriles, náuseas, vómitos y reacciones anafilácticas. 
Además puede transmitir virus $\mathrm{y}$ priones por ser de origen biológico. El hidroxietilalmidón $6 \%$ es más barato, y seguro para la prevención y tratamiento del síndrome. Tampoco está claro el momento exacto de la administración ni si se debiera repetir la misma para evitar los cuadros tardíos.

7. Administración profiláctica de corticoides: su uso también es controvertido. Teóricamente podrían ser útiles por su efecto antiinflamatorio.

8. Agonistas dopaminérgicos ${ }^{11}$ : continúan en investigación. Actúan inactivando el receptor-2 del VEGF, con lo que inhibirían el proceso fisiopatológico.

9. Coasting: administrar gonadotropinas y administrar agonistas GnRH de forma discontínua mientras se realizan controles por ecografía y analítica, hasta que el estradiol baje a cifras de seguridad. Es un método muy empleado aunque aún son precisos más estudios para comprobar su utilidad real. Parece que aunque diariamente se pierdan folículos y la calidad de los ovocitos sea menor, la espera de entre 2-6 días no disminuye la tasa de embarazos.

10. Aspiración folicular temprana unilateral (a las 10-12h de la hCG); su utilidad no se ha demostrado. Sin embargo, la punción y aspiración meticulosa de todos los folículos estimulados cuando se hace recuperación ovocitaria, podría interferir en los mecanismos desencadenantes y mediadores del SHO disminuyendo su aparición.

\section{BIBLIOGRAFÍA}

1. PROSEGO: Protocolos y guías de actualización clínica en Ginecología y Obstetricia. Síndrome de hiperestimulación ovárica (Protocolo actualizado en 2005). http: www. prosego.com.

2. Speroff L, Fritz MA. Inducción de la ovulación, síndrome de hiperestimulación ovárica. En: Endocrinología Ginecológica Clínica y Esterilidad $7^{\mathrm{a}}$ Edición. Eds Wolters Kluwer Health España, S.A. 2006; 31: 1198-1200.

3. García-Velasco JA, Pellicer A. New concepts in the understanding of the ovarian hyperstimulation syndrome. Curr Opin Obstet Gynecol 2003; 15: 251-256.

4. Whelan JG, Vlahos NF. The ovarian hyperstimulation syndrome. Fertil Steril 2000; 73: 883-896.

5. Delvinge A, Rozenberg S. Review of clinical course and treatment of ovarian hyperstimulation syndrome (OHSS). Hum Reprod Update 2003; 9: 77-96.

6. Practice committee of american society for reproductive medicine. Ovarian hyperstimulation syndrome. Rev Fertil Steril 2008; 90 (Suppl 5): 188-193.

7. Royal college of obstetricians and gynaecologists. green-top guideline The management of ovarian hyperstimulation syndrome. No. 5. Sept 2006, 1-11. http://www.rcog.org.uk/.

8. COHEN BM. Role of human albumin in ovarian hyperstimulation syndrome. Fertil Steril 2008; 89:1845-1846.

9. Aboulghar MA, Mansour RT. Ovarian hyperstimulation syndrome: calsifications and critical analysis of preventive measures. Review. Human Reprod Update 2003; 9: 275-289.

10. Delvinge A, Rozenberg S. Epidemiology and prevention of ovarian hyperstimulation syndrome (OHSS): a review. Hum Reprod Update $2002 ; 6$ : 559-577.

11. Alvárez C, Martí-Bonmatí L, Novella-Maestre E, Sanz R, Gómez R, Fernández M et al. Dopamine agonist cabergoline reduces hemoconcentration and ascitis in hyperstimulated women undergoing assisted reproduction. J Clin Endocrinol Metab 2007; 92: 2931-2937. 
